\title{
General expressions of the second-order approximation of the Chapman-Enskog method for the Enskog-Landau kinetic equation
}

\author{
M.V.Tokarchuk, Y.A.Humenyuk \\ Institute for Condensed Matter Physics \\ of the National Academy of Sciences of Ukraine, \\ 1 Svientsitskii Str., 79011 Lviv, Ukraine
}

Received October 13, 2000, in final form December 4, 2000

\begin{abstract}
The second-order approximation of the Chapman-Enskog method is considered for the Enskog-Landau kinetic equation. Exact expressions for the pair distribution function of the nonuniform hard-sphere system are presented up to the second order in gradients. A general equation for the second-order correction to the one-particle distribution function is obtained. Corresponding contributions to momentum and heat fluxes are found.
\end{abstract}

Key words: kinetic equation, Chapman-Enskog method, second-order correction

PACS: 05.20.Dd, 05.60.-k, 52.25.Fi

\section{Introduction}

Nonlinear processes in ionized dense gases and plasmas are a subject of both experimental and theoretical interest. Higher-order gradients of the local number density, temperature, and average velocity are characteristic of them. Moreover, the cross correlations between thermo-diffusion, viscous, and heat processes are important. Such processes can be investigated on the basis of kinetic equations within higher approximations on gradient expansions in the Chapman-Enskog method [1].

Solving a kinetic equation by means of the Chapman-Enskog method, the oneparticle distribution function is searched as an expansion in gradients of the hydrodynamic quantities (number density, average velocity and temperature). As a rule consideration is restricted only to the first approximation for the one-particle distribution function. The first-order correction contains terms linear in the gradients of the hydrodynamic quantities. As a result one obtains the Navier-Stokes hydrodynamic equations.

The Chapman-Enskog method also permits to find the next corrections to the 
distribution function, which contain high-order gradients and gradient products. The second correction $f^{2}$ for the Boltzmann kinetic equation was considered by Burnett [1] and the second-order contributions to pressure tensor and heat flux vector were found. Finally, new transport equations, the so-called Burnett equations, were obtained.

The possibility of considering the second-order correction for the Enskog kinetic equation has been discussed by van Beijeren et al. [2]. The authors of this work showed that the usual Enskog equation does not properly take into account the second-order and higher terms, and this problem should be studied starting from the modified Enskog equation proposed by them.

Since the Enskog kinetic equation is more complicated in structure than the Boltzmann equation, the second-order correction to the one-particle distribution function may be expected to involve new terms, not arising in the case of the Boltzmann equation, and this is also true for the second-order contributions to the pressure tensor and heat flux vector. It is precisely these aspects that are the subject of our study.

We shall consider the second-order correction $f^{2}$ for the Enskog-Landau kinetic equation [3] in the framework of the Chapman-Enskog method. All the results for the modified Enskog equation can be obtained as a particular case.

In section 2 we present the Enskog-Landau kinetic equation for the one-component system of charged hard spheres, write down conservation laws obtained from this equation, and also give general expressions for momentum and heat fluxes. In section 3 the gradient expansion for the pair distribution function is considered and explicit second-order terms are calculated. The equations of various orders for the corrections to the one-particle distribution function are written in section 4 . In section 5 we find the contributions of various orders to the fluxes. Some conclusive remarks are made in section 6.

\section{Kinetic equation, conservation laws and fluxes}

The Enskog-Landau kinetic equation was obtained from the BBGKY hierarchy of equations with modified boundary conditions in the pair collision approximation [3]. It describes a one-component system of charged hard spheres in a compensating field. The interparticle interaction potential consists of the short-range part (hard spheres) and the long-range tail (Coulomb interaction), and can be written as

$$
\Phi\left(r_{21}\right)=\left\{\begin{array}{cc}
\infty, & r_{21}<\sigma \\
\Phi_{t}\left(r_{21}\right), & r_{21}>\sigma
\end{array}\right.
$$

where $\sigma$ denotes the hard-sphere diameter, $\Phi_{t}\left(r_{21}\right)=e^{2} / r_{21}$.

The Enskog-Landau kinetic equation is of the form:

$$
\left(\partial_{t}+\boldsymbol{v}_{1} \cdot \nabla_{1}\right) f\left(x_{1}, t\right)=I_{\mathrm{E}}(f, f)+I_{\mathrm{MF}}(f, f)+I_{\mathrm{L}}(f, f) \equiv I(f, f),
$$


with $\partial_{t} \equiv \frac{\partial}{\partial t}, \nabla_{1} \equiv \frac{\partial}{\partial \boldsymbol{r}_{1}}, x_{1}=\left\{\boldsymbol{r}_{1}, \boldsymbol{v}_{1}\right\}$. The three terms in the right-hand side describe contributions due to both parts of the interaction potential $\Phi\left(r_{21}\right)$.

$$
\begin{aligned}
& I_{\mathrm{E}}(f, f)=\sigma^{2} \int \mathrm{d} \boldsymbol{v}_{2} \mathrm{~d} \hat{\sigma} \theta\left(\hat{\sigma} \cdot \boldsymbol{v}_{21}\right) \hat{\sigma} \cdot \boldsymbol{v}_{21} \\
& \quad \times\left[g_{2}\left(\boldsymbol{r}_{1}, \boldsymbol{r}_{1}+\boldsymbol{\sigma}, t\right) f\left(\boldsymbol{r}_{1}, \boldsymbol{v}_{1}^{\prime}, t\right) f\left(\boldsymbol{r}_{1}+\boldsymbol{\sigma}, \boldsymbol{v}_{2}^{\prime}, t\right)\right. \\
& \left.\quad-g_{2}\left(\boldsymbol{r}_{1}, \boldsymbol{r}_{1}-\boldsymbol{\sigma}, t\right) f\left(\boldsymbol{r}_{1}, \boldsymbol{v}_{1}, t\right) f\left(\boldsymbol{r}_{1}-\boldsymbol{\sigma}, \boldsymbol{v}_{2}, t\right)\right]
\end{aligned}
$$

is the collision integral of the revised Enskog theory (RET) [2]; $\hat{\sigma}$ indicates a unit vector, $\theta$ is the Heaviside step function, $\boldsymbol{v}_{21}=\boldsymbol{v}_{2}-\boldsymbol{v}_{1}, g_{2}$ is the pair distribution function, $\boldsymbol{\sigma}=\sigma \hat{\sigma}$, primes on velocities denote postcollisional values.

$$
I_{\mathrm{MF}}(f, f)=-\frac{1}{m} \frac{\partial}{\partial \boldsymbol{v}_{1}} f\left(x_{1}, t\right) \cdot \int \mathrm{d} \boldsymbol{r}_{2} \hat{r}_{21} \Phi_{t}^{\prime}\left(r_{21}\right) n\left(\boldsymbol{r}_{2}, t\right) g_{2}\left(\boldsymbol{r}_{1}, \boldsymbol{r}_{2}, t\right),
$$

where $\hat{r}_{21}=\boldsymbol{r}_{21} / r_{21}, r_{21}=\left|\boldsymbol{r}_{21}\right|, \Phi_{t}^{\prime}\left(r_{21}\right)=\mathrm{d} \Phi_{t} / \mathrm{d} r_{21} . I_{\mathrm{MF}}$ is the collision integral of the kinetic mean-field theory (KMFT) [4], it is of the first order in the long-range part $\Phi_{t}\left(r_{21}\right)$ of the interaction potential.

$$
I_{\mathrm{L}}(f, f)=\int \mathrm{d} \boldsymbol{v}_{2} \mathrm{~d} b \mathrm{~d} \epsilon b v_{21}\left[f\left(\boldsymbol{r}_{1}, \boldsymbol{v}_{1}^{*}, t\right) f\left(\boldsymbol{r}_{1}, \boldsymbol{v}_{2}^{*}, t\right)-f\left(\boldsymbol{r}_{1}, \boldsymbol{v}_{1}, t\right) f\left(\boldsymbol{r}_{1}, \boldsymbol{v}_{2}, t\right)\right]
$$

is the so-called Landau-like collision integral [3], which is of the second order in the long-range part $\Phi_{t}\left(r_{21}\right)$. Here, it is written in the simplified Boltzmann-like form within the approximation $g_{2}\left(\boldsymbol{r}_{1}, \boldsymbol{r}_{2}, t\right) \rightarrow 1$. This approximation is mathematically convenient only, but is not necessary. $b$ is the impact parameter, $\epsilon$ is the azimuthal angle of scattering, $\boldsymbol{v}_{1}^{*}=\boldsymbol{v}_{1}-\frac{1}{2} \triangle \boldsymbol{v}_{21}, \boldsymbol{v}_{2}^{*}=\boldsymbol{v}_{2}+\frac{1}{2} \triangle \boldsymbol{v}_{21}$ are velocities of the particles 1 and 2 after the Coulomb scattering, where

$$
\triangle \boldsymbol{v}_{21}=-\left.\frac{1}{m^{*} v_{21}} \int_{-\infty}^{+\infty} \mathrm{d} \xi \frac{\partial \Phi_{t}\left(r_{21}\right)}{\partial \boldsymbol{r}_{21}}\right|_{r_{21}=\sqrt{\xi^{2}+b^{2}}}
$$

is the change of the relative velocity vector $\boldsymbol{v}_{21}$ associated with the Coulomb scattering of the particles, and $m^{*}=m_{1} m_{2} /\left(m_{1}+m_{2}\right)=m / 2$ is the reduced mass.

The local number density, average velocity, and temperature are defined by

$$
\begin{aligned}
n\left(\boldsymbol{r}_{1}, t\right) & =\int \mathrm{d} \boldsymbol{v}_{1} f\left(x_{1}, t\right), \\
\rho\left(\boldsymbol{r}_{1}, t\right) \boldsymbol{V}\left(\boldsymbol{r}_{1}, t\right) & =\int \mathrm{d} \boldsymbol{v}_{1} f\left(x_{1}, t\right) m \boldsymbol{v}_{1}, \\
\frac{3}{2} n\left(\boldsymbol{r}_{1}, t\right) k T\left(\boldsymbol{r}_{1}, t\right) & =\int \mathrm{d} \boldsymbol{v}_{1} f\left(x_{1}, t\right) \frac{m}{2}\left(\boldsymbol{v}_{1}-\boldsymbol{V}\right)^{2},
\end{aligned}
$$

where $\rho\left(\boldsymbol{r}_{1}, t\right)=m n\left(\boldsymbol{r}_{1}, t\right)$. We can obtain conservation laws for these quantities from the kinetic equation (1) by multiplying it by $\psi\left(\boldsymbol{v}_{1}\right)=\left\{1, m \boldsymbol{v}_{1}, m v_{1}^{2} / 2\right\}$ and integrating over $\boldsymbol{v}_{1}$ :

$$
\begin{aligned}
& \partial_{t} n+\nabla_{1} \cdot(n \boldsymbol{V})=0, \\
& \partial_{t}(\rho \boldsymbol{V})+\nabla_{1} \cdot\left(\mathrm{P}^{\mathrm{k}}+\rho \boldsymbol{V} \boldsymbol{V}\right)=-\nabla_{1} \cdot\left(\mathrm{P}^{\mathrm{t}}+\mathrm{P}^{\mathrm{hs}}\right), \\
& \begin{array}{c}
\partial_{t}\left(\frac{3}{2} n k T+\frac{\rho}{2} V^{2}\right)+\nabla_{1} \cdot\left(\boldsymbol{q}^{\mathrm{k}}+\boldsymbol{V} \cdot \mathrm{P}^{\mathrm{k}}+\frac{3}{2} n k T \boldsymbol{V}+\frac{\rho}{2} \boldsymbol{V} V^{2}\right)= \\
\quad=-\boldsymbol{V} \cdot\left(\nabla_{1} \cdot \mathrm{P}^{\mathrm{t}}\right)-\nabla_{1} \cdot\left(\boldsymbol{q}^{\mathrm{hs}}+\boldsymbol{V} \cdot \mathrm{P}^{\mathrm{hs}}\right) .
\end{array}
\end{aligned}
$$


Expressions for the various types of contributions to the momentum and heat fluxes are given by

$$
\left(\begin{array}{c}
\mathrm{P}^{\mathrm{k}} \\
\boldsymbol{q}^{\mathrm{k}}
\end{array}\right)=\int \mathrm{d} \boldsymbol{v}_{1} f\left(x_{1}, t\right)\left(\begin{array}{c}
m \boldsymbol{c}_{1} \boldsymbol{c}_{1} \\
\frac{m}{2} c_{1}^{2} \boldsymbol{c}_{1}
\end{array}\right)
$$

where $\boldsymbol{c}_{1}=\boldsymbol{v}_{1}-\boldsymbol{V}$

$$
\mathbf{P}^{\mathrm{t}}=-\frac{1}{2} \int \mathrm{d} \boldsymbol{s} \boldsymbol{s} \hat{s} \Phi_{t}^{\prime}(s) \int_{0}^{1} \mathrm{~d} \lambda n_{2}\left(\boldsymbol{r}_{1}+\lambda \boldsymbol{s}, \boldsymbol{r}_{1}+(\lambda-1) \boldsymbol{s}\right) .
$$

Here $\hat{s}=\boldsymbol{s} / s, n_{2}\left(\boldsymbol{r}_{1}, \boldsymbol{r}_{2}\right)=n\left(\boldsymbol{r}_{1}, t\right) n\left(\boldsymbol{r}_{2}, t\right) g_{2}\left(\boldsymbol{r}_{1}, \boldsymbol{r}_{2}, t\right)$.

$$
\begin{aligned}
\left(\begin{array}{c}
\mathrm{Phs}^{\mathrm{hs}} \\
\boldsymbol{q}^{\mathrm{hs}}
\end{array}\right)= & \frac{\sigma^{3}}{2} \int \mathrm{d} \boldsymbol{v}_{1} \mathrm{~d} \boldsymbol{v}_{2} \mathrm{~d} \hat{\sigma} \theta\left(\hat{\sigma} \cdot \boldsymbol{v}_{21}\right) \hat{\sigma} \cdot \boldsymbol{v}_{21}\left(\begin{array}{c}
m\left[\boldsymbol{v}_{1}^{\prime}-\boldsymbol{v}_{1}\right] \\
\frac{m}{2}\left[c_{1}^{\prime 2}-c_{1}^{2}\right]
\end{array}\right) \hat{\sigma} \\
& \times \int_{0}^{1} \mathrm{~d} \lambda f_{2}\left(\boldsymbol{r}_{1}+\lambda \boldsymbol{\sigma}, \boldsymbol{v}_{1}, \boldsymbol{r}_{1}+(\lambda-1) \boldsymbol{\sigma}, \boldsymbol{v}_{2}, t\right),
\end{aligned}
$$

where $f_{2}\left(\boldsymbol{r}_{1}, \boldsymbol{v}_{1}, \boldsymbol{r}_{2}, \boldsymbol{v}_{2}, t\right)=g_{2}\left(\boldsymbol{r}_{1}, \boldsymbol{r}_{2}, t\right) f\left(\boldsymbol{r}_{1}, \boldsymbol{v}_{1}\right) f\left(\boldsymbol{r}_{2}, \boldsymbol{v}_{2}\right)$.

The kinetic (or streaming) contributions are denoted by the superscript $k$, the contribution arising from the $I_{\mathrm{MF}}$ term (tail term) is denoted by the superscript $t$, and the superscript $h s$ means the collisional contributions. $I_{\mathrm{L}}$ does not contribute to the fluxes due to its Boltzmann-like form, but an additional long-range term arises in $\Omega$-integrals because of $I_{\mathrm{L}}$.

Equations (5) and (6) are simplified, when $\partial_{t} n$ and $\partial_{t} \boldsymbol{V}$ are eliminated. We obtain them in the form:

$$
\begin{aligned}
& {\left[\partial_{t}+\boldsymbol{V} \cdot \nabla_{1}\right] \boldsymbol{V}+\frac{1}{\rho} \nabla_{1} \cdot \mathrm{P}=0,} \\
& n\left[\partial_{t}+\boldsymbol{V} \cdot \nabla_{1}\right] \frac{3}{2} k T+\nabla_{1} \cdot \boldsymbol{q}+\mathrm{P}_{*}: \nabla_{1} \boldsymbol{V}=0,
\end{aligned}
$$

where $\mathrm{P}=\mathrm{P}^{\mathrm{k}}+\mathrm{P}^{\mathrm{hs}}+\mathrm{P}^{\mathrm{t}}, \mathrm{P}_{*}=\mathrm{P}^{\mathrm{k}}+\mathrm{P}^{\mathrm{hs}}$, and $\boldsymbol{q}=\boldsymbol{q}^{\mathrm{k}}+\boldsymbol{q}^{\mathrm{hs}}$.

\section{Gradient expansion of $g_{2}$}

The pair distribution function $g_{2}$ in the collision integrals $I_{\mathrm{E}}$ and $I_{\mathrm{MF}}$ takes into account the full interaction potential (the hard-sphere part plus the long-range tail). Thus it is a functional of the local number density $n(\boldsymbol{r}, t)$ and the local temperature $T(\boldsymbol{r}, t)[5]$. But we shall use for $g_{2}$ in our calculation the following approximation:

$$
g_{2}\left(\boldsymbol{r}_{1}, \boldsymbol{r}_{2}, t\right) \approx g_{2}^{\mathrm{hs}}\left(\boldsymbol{r}_{1}, \boldsymbol{r}_{2} \mid n\right)
$$

where $g_{2}^{\text {hs }}\left(\boldsymbol{r}_{1}, \boldsymbol{r}_{2} \mid n\right)$ is the form assumed by $g_{2}$ for a hard-sphere system (i.e. for $\left.\Phi_{t}\left(r_{21}\right) \equiv 0\right)$ in inhomogeneous equilibrium with number density $n(\boldsymbol{r}, t)$. This is the so-called kinetic-variational theory approximation [4]. Thus $g_{2}^{\text {hs }}$ (which however will be denoted further as $g_{2}$ ) is considered as a nonlocal functional of the local number density $n(\boldsymbol{r}, t)[2]$. In this section we give the gradient expansion of $g_{2}$ up 
to the second order within the approximation (12). We shall present corresponding expressions in a general case for any mutual dispositions of particles 1 and 2 .

Let $\boldsymbol{r}_{p}, \boldsymbol{r}_{q}$ denote coordinates of centres of the particles 1 and 2 , and we want to expand $g_{2}\left(\boldsymbol{r}_{p}, \boldsymbol{r}_{q} \mid n\right)$ in gradients calculated at position $\boldsymbol{r}_{e}$. We can write for $g_{2}$ a functional series:

$$
\begin{aligned}
& g_{2}\left(\boldsymbol{r}_{p}, \boldsymbol{r}_{q} \mid n\right)=g_{2}^{0}\left(\boldsymbol{r}_{p}, \boldsymbol{r}_{q} \mid n, \boldsymbol{r}_{e}\right)+\delta \int \mathrm{d} \boldsymbol{r}_{s} H^{1}\left(\boldsymbol{r}_{p}, \boldsymbol{r}_{q} ; \boldsymbol{r}_{s} \mid n, \boldsymbol{r}_{e}\right) \nabla_{e} n\left(\boldsymbol{r}_{e}\right) \cdot \boldsymbol{r}_{s e} \\
& \quad+\delta^{2} \frac{1}{2} \int \mathrm{d} \boldsymbol{r}_{s} H^{1}\left(\boldsymbol{r}_{p}, \boldsymbol{r}_{q} ; \boldsymbol{r}_{s} \mid n, \boldsymbol{r}_{e}\right) \nabla_{e} \nabla_{e} n\left(\boldsymbol{r}_{e}\right): \boldsymbol{r}_{s e} \boldsymbol{r}_{s e} \\
& \quad+\delta^{2} \frac{1}{2} \int \mathrm{d} \boldsymbol{r}_{s} d \boldsymbol{r}_{v} H^{2}\left(\boldsymbol{r}_{p}, \boldsymbol{r}_{q} ; \boldsymbol{r}_{s}, \boldsymbol{r}_{v} \mid n, \boldsymbol{r}_{e}\right) \nabla_{e} n\left(\boldsymbol{r}_{e}\right) \nabla_{e} n\left(\boldsymbol{r}_{e}\right): \boldsymbol{r}_{s e} \boldsymbol{r}_{v e}+\ldots \equiv \\
& \quad \equiv g_{2}^{0}\left(\boldsymbol{r}_{p}, \boldsymbol{r}_{q} \mid n, \boldsymbol{r}_{e}\right)+\delta g_{2}^{1}\left(\boldsymbol{r}_{p}, \boldsymbol{r}_{q} \mid n, \boldsymbol{r}_{e}\right)+\delta^{2} g_{2}^{2}\left(\boldsymbol{r}_{p}, \boldsymbol{r}_{q} \mid n, \boldsymbol{r}_{e}\right)+\ldots
\end{aligned}
$$

The parameter $\delta$ is formal. It denotes term orders in the gradient expansion. The functions $H^{j}$ are functional derivatives of $g_{2}$ with respect to $n\left(\boldsymbol{r}_{s}\right), n\left(\boldsymbol{r}_{v}\right)$, and so on, evaluated at the uniform number density, which is equal to $n\left(\boldsymbol{r}_{e}\right)$.

We need explicit expressions for the terms in the right-hand side of equation (13). In [2] the term $\sim \delta$ has been considered and found using symmetry properties of the function $H^{1}$ in a Cartesian frame of reference. But in general, it is convenient to consider the terms $\sim \delta$ or $\sim \delta^{2}$ in a bipolar system of coordinates [6] with poles situated at the positions $\boldsymbol{r}_{p}$ and $\boldsymbol{r}_{q}$. At first, it is necessary to write the terms of equation (13) in the bipolar system of coordinates, afterwards one should perform possible integrations and come back to the Cartesian frame of reference. In the appendix we give some calculation details of the next expressions. The terms $g_{2}^{0}, g_{2}^{1}$, and $g_{2}^{2}$ have the following form:

$$
\begin{aligned}
g_{2}^{0}\left(\boldsymbol{r}_{p}, \boldsymbol{r}_{q} \mid n, \boldsymbol{r}_{e}\right) & =g_{2}^{\mathrm{eq}}\left(\boldsymbol{r}_{p}, \boldsymbol{r}_{q} \mid n, \boldsymbol{r}_{e}\right), \\
g_{2}^{1}\left(\boldsymbol{r}_{p}, \boldsymbol{r}_{q} \mid n, \boldsymbol{r}_{e}\right) & =\nabla_{e} n \cdot\left[\frac{1}{2} \boldsymbol{r}_{q p}+\boldsymbol{r}_{p e}\right] \tau_{0}, \\
g_{2}^{2}\left(\boldsymbol{r}_{p}, \boldsymbol{r}_{q} \mid n, \boldsymbol{r}_{e}\right) & =\frac{1}{2} \nabla_{e} \nabla_{e} n:\left(\nu \mathbf{I}+(\mu-\nu) \hat{r}_{q p} \hat{r}_{q p}\right. \\
& \left.+\tau_{0}\left[\frac{1}{2}\left(\boldsymbol{r}_{q p} \boldsymbol{r}_{p e}+\boldsymbol{r}_{p e} \boldsymbol{r}_{q p}\right)+\boldsymbol{r}_{p e} \boldsymbol{r}_{p e}\right]\right) \\
& +\frac{1}{2} \nabla_{e} n \nabla_{e} n:\left(\nu_{2} \mathbf{I}+\left(\mu_{2}-\nu_{2}\right) \hat{r}_{q p} \hat{r}_{q p}\right. \\
& \left.+\partial_{n} \tau_{0}\left[\frac{1}{2}\left(\boldsymbol{r}_{q p} \boldsymbol{r}_{p e}+\boldsymbol{r}_{p e} \boldsymbol{r}_{q p}\right)+\boldsymbol{r}_{p e} \boldsymbol{r}_{p e}\right]\right) .
\end{aligned}
$$

In equation (14) $g_{2}^{\mathrm{eq}}\left(\boldsymbol{r}_{p}, \boldsymbol{r}_{q} \mid n, \boldsymbol{r}_{e}\right)$ is the equilibrium pair distribution function for the hard-sphere system with the uniform number density $n$ being equal to the value of the local number density at position $\boldsymbol{r}_{e}$. The coefficients are defined by

$$
\begin{aligned}
& \nu=-\frac{r_{q p}^{2}}{8} \tau_{0}+\frac{1}{2} \tau_{2}-\frac{1}{4 r_{q p}^{2}} \tau_{3}, \\
& \mu=\frac{r_{q p}^{2}}{4} \tau_{0}+\frac{1}{2 r_{q p}^{2}} \tau_{3},
\end{aligned}
$$

where $\tau_{0}, \tau_{2}, \tau_{3}$ are 'moments' of the function $H^{1}$ and in the Cartesian frame of reference they read:

$$
\tau_{0}=\int \mathrm{d} \boldsymbol{r}_{s} H^{1}\left(\boldsymbol{r}_{p}, \boldsymbol{r}_{q} ; \boldsymbol{r}_{s} \mid n, \boldsymbol{r}_{e}\right)=\partial_{n} g_{2}^{0}\left(\boldsymbol{r}_{p}, \boldsymbol{r}_{q} \mid n, \boldsymbol{r}_{e}\right)
$$




$$
\begin{aligned}
& \tau_{2}=\int \mathrm{d} \boldsymbol{r}_{s} H^{1}\left(\boldsymbol{r}_{p}, \boldsymbol{r}_{q} ; \boldsymbol{r}_{s} \mid n, \boldsymbol{r}_{e}\right) r_{s p}^{2}, \\
& \tau_{3}=\int \mathrm{d} \boldsymbol{r}_{s} H^{1}\left(\boldsymbol{r}_{p}, \boldsymbol{r}_{q} ; \boldsymbol{r}_{s} \mid n, \boldsymbol{r}_{e}\right) r_{s p}^{2}\left[r_{s p}^{2}-r_{s q}^{2}\right],
\end{aligned}
$$

with $\partial_{n}=\frac{\partial}{\partial n}$. The other two coefficients are:

$$
\begin{aligned}
\mu_{2} & =\frac{r_{q p}^{2}}{4} \alpha_{0}+\frac{1}{2} \alpha_{2}+\frac{1}{2 r_{q p}^{2}} \alpha_{3} \\
\nu_{2} & =-\frac{\pi}{4 r^{2}} \int_{0}^{\infty} \mathrm{d} s \int_{|r-s|}^{r+s} \mathrm{~d} t \frac{s t}{r} \int_{0}^{\infty} \mathrm{d} s_{1} \int_{\left|r-s_{1}\right|}^{r+s_{1}} \mathrm{~d} t_{1} \frac{s_{1} t_{1}}{r} \int_{0}^{2 \pi} \mathrm{d} \chi \\
& \times \xi(r, s, t) \xi\left(r, s_{1}, t_{1}\right) H^{2}\left(r,\left[\begin{array}{c}
s, s_{1} \\
t, t_{1}
\end{array}\right], \chi\right) \cos \chi
\end{aligned}
$$

The coefficients $\alpha_{0}, \alpha_{2}, \alpha_{3}$ are 'moments' of $H^{2}$ which in the Cartesian frame of reference read:

$$
\begin{aligned}
& \alpha_{0}=\int \mathrm{d} \boldsymbol{r}_{s} \boldsymbol{r}_{v} H^{2}\left(\boldsymbol{r}_{p}, \boldsymbol{r}_{q}, \boldsymbol{r}_{s}, \boldsymbol{r}_{v} \mid n, \boldsymbol{r}_{e}\right)=\partial_{n} \partial_{n} g_{2}^{0}\left(\boldsymbol{r}_{p}, \boldsymbol{r}_{q} \mid n, \boldsymbol{r}_{e}\right)=\partial_{n} \tau_{0}, \\
& \alpha_{2}=\int \mathrm{d} \boldsymbol{r}_{s} \boldsymbol{r}_{v} H^{2}\left(\boldsymbol{r}_{p}, \boldsymbol{r}_{q}, \boldsymbol{r}_{s}, \boldsymbol{r}_{v} \mid n, \boldsymbol{r}_{e}\right)\left[r_{s p}^{2}-r_{s q}^{2}\right], \\
& \alpha_{3}=\int \mathrm{d} \boldsymbol{r}_{s} \boldsymbol{r}_{v} H^{2}\left(\boldsymbol{r}_{p}, \boldsymbol{r}_{q}, \boldsymbol{r}_{s}, \boldsymbol{r}_{v} \mid n, \boldsymbol{r}_{e}\right) r_{s p}^{2}\left[r_{v p}^{2}-r_{v q}^{2}\right],
\end{aligned}
$$

where $r=r_{q p}, s=r_{s p}, t=r_{s q}, s_{1}=r_{v p}, t_{1}=r_{v q}, \chi$ is an angle between projections of the vectors $\boldsymbol{r}_{s p}$ and $\boldsymbol{r}_{s v}$ on a plane normal to the direction $\boldsymbol{r}_{q p}$. $\xi(r, s, t)=\sqrt{\left[t^{2}-(s-r)^{2}\right]\left[(s+r)^{2}-t^{2}\right]}$ and $\xi\left(r, s_{1}, t_{1}\right)$ is the same function of $s_{1}$ and $t_{1}$.

The term $g_{2}^{1}$ is given in [2], but we have calculated it in a general case. Besides, it can be seen from equation (15) that when we expand $g_{2}$ in gradients calculated at the position $\boldsymbol{r}_{e}=\left(\boldsymbol{r}_{p}+\boldsymbol{r}_{q}\right) / 2$, term $g_{2}^{1}$ vanishes. This is one of the results of [2] which establishes the equivalence between the usual Enskog equation and the modified equation when only the first-order terms are considered.

The $g_{2}^{2}$ is more complicated. The tensorial structure of both terms proportional to $\nabla_{e} \nabla_{e} n$ and $\nabla_{e} n \nabla_{e} n$ turned out to be the same. We also have the explicit dependence of $g_{2}^{2}$ on $\hat{r}_{q p}$, which we shall need further in our particular calculations.

In the table below we list quantities, for which formulae (14)-(16) for $g_{2}^{j}$ are used, and corresponding values of $\boldsymbol{r}_{p}, \boldsymbol{r}_{q}$, and $\boldsymbol{r}_{e}$ for substitutions.

\begin{tabular}{l|llll} 
& in $I_{\mathrm{E}}^{j}$ & in $\mathrm{P}^{h s(j)}$ and $\boldsymbol{q}^{h s(j)}$ & in $\boldsymbol{i}_{\mathrm{MF}}^{j}$ & in $\mathrm{P}^{t(j)}$ \\
\hline $\boldsymbol{r}_{p}$ & $\boldsymbol{r}_{1}$ & $\boldsymbol{r}_{1}+\lambda \boldsymbol{\sigma}$ & $\boldsymbol{r}_{1}$ & $\boldsymbol{r}_{1}+\lambda \boldsymbol{s}$ \\
$\boldsymbol{r}_{q}$ & $\boldsymbol{r}_{1} \pm \boldsymbol{\sigma}$ & $\boldsymbol{r}_{1}+(\lambda-1) \boldsymbol{\sigma}$ & $\boldsymbol{r}_{2}$ & $\boldsymbol{r}_{1}+(\lambda-1) \boldsymbol{s}$ \\
$\boldsymbol{r}_{e}$ & $\boldsymbol{r}_{1}$ & $\boldsymbol{r}_{1}$ & $\boldsymbol{r}_{1}$ & $\boldsymbol{r}_{1}$ \\
$\boldsymbol{r}_{q p}$ & $\pm \boldsymbol{\sigma}$ & $-\boldsymbol{\sigma}$ & $\boldsymbol{r}_{21}$ & $-\boldsymbol{s}$ \\
$\hat{r}_{q p}$ & $\pm \hat{\sigma}$ & $-\hat{\sigma}$ & $\hat{r}_{21}$ & $-\hat{s}$ \\
$\boldsymbol{r}_{p e}$ & 0 & $\lambda \boldsymbol{\sigma}$ & 0 & $\lambda \boldsymbol{s}$
\end{tabular}


Now we want to compare the obtained formulae (14)-(16) for the functional $g_{2}$ with the corresponding formulae for the equilibrium pair distribution function $g_{2}^{\text {eq }}$ of the hard-sphere system with a uniform number density, which equals the local number density $n\left(\left[\boldsymbol{r}_{1}+\boldsymbol{r}_{2}\right] / 2\right)$ in the point of contact of the particles 1 and 2 . Let $\boldsymbol{r}_{p}, \boldsymbol{r}_{q}, \boldsymbol{r}_{e}$ denote the same as before, and let us denote $\left[\boldsymbol{r}_{p}+\boldsymbol{r}_{q}\right] / 2=\boldsymbol{r}_{c}$. For $g_{2}^{\mathrm{eq}}\left(\boldsymbol{r}_{p}, \boldsymbol{r}_{q} \mid n, \boldsymbol{r}_{c}\right)$ we can write a series:

$$
g_{2}^{\mathrm{eq}}\left(\boldsymbol{r}_{p}, \boldsymbol{r}_{q} \mid n, \boldsymbol{r}_{c}\right)=\left[1+\delta \boldsymbol{r}_{c e} \cdot \nabla_{e}+\delta^{2} \frac{1}{2} \boldsymbol{r}_{c e} \boldsymbol{r}_{c e}: \nabla_{e} \nabla_{e}+\ldots\right] g_{2}^{\mathrm{eq}}\left(\boldsymbol{r}_{p}, \boldsymbol{r}_{q} \mid n, \boldsymbol{r}_{e}\right),
$$

where $\boldsymbol{r}_{c e}=\boldsymbol{r}_{c}-\boldsymbol{r}_{e}$. Substituting $\boldsymbol{r}_{c e}=\boldsymbol{r}_{c p}+\boldsymbol{r}_{p e}$ and the value of $\boldsymbol{r}_{c}$ into equation (21) we get a gradient expansion for $g_{2}^{\mathrm{eq}}\left(\boldsymbol{r}_{p}, \boldsymbol{r}_{q} \mid n, \boldsymbol{r}_{c}\right)$ :

$$
\begin{aligned}
& g_{2}^{\mathrm{eq}}\left(\boldsymbol{r}_{p}, \boldsymbol{r}_{q} \mid n, \boldsymbol{r}_{c}\right)=\left[1+\delta\left(\frac{1}{2} \boldsymbol{r}_{q p}+\boldsymbol{r}_{p e}\right) \cdot \nabla_{e} n \partial_{n}+\delta^{2} \frac{1}{2}\left(\frac{1}{4} \boldsymbol{r}_{q p} \boldsymbol{r}_{q p}+\frac{1}{2}\left[\boldsymbol{r}_{q p} \boldsymbol{r}_{p e}\right.\right.\right. \\
& \left.\left.\left.\quad+\boldsymbol{r}_{p e} \boldsymbol{r}_{q p}\right]+\boldsymbol{r}_{p e} \boldsymbol{r}_{p e}\right):\left\{\nabla_{e} \nabla_{e} n \partial_{n}+\nabla_{e} n \nabla_{e} n \partial_{n} \partial_{n}\right\}+\ldots\right] g_{2}^{\mathrm{eq}}\left(\boldsymbol{r}_{p}, \boldsymbol{r}_{q} \mid n, \boldsymbol{r}_{e}\right) .
\end{aligned}
$$

It can be seen that the discrepancy between equations (14)-(16) and (22) arises in the second-order terms. It lies in the distinction between the coefficients at the tensor $\hat{r}_{q p} \hat{r}_{q p}$ and there are additional terms with the unit tensor I in equation (16).

The latter comment concerns numerical calculations. One can see that the firstorder correction for $g_{2}$ includes the usual derivative $\partial_{n} g_{2}^{\text {eq }}$, though it is known to be unnecessary for calculation of the transport coefficients [1]. By contrast to this case, to calculate the second-order contributions to $\mathrm{P}$ and $\boldsymbol{q}$ numerically, one needs not only to know the second derivative $\partial_{n} \partial_{n} g_{2}^{\text {eq }}$ but also the first and the second functional derivatives of the functional $g_{2}$ (more precisely, their certain 'moments' are needed).

\section{Expansion of collision integrals and equations of various orders}

Before we apply the Chapman-Enskog method to the Enskog-Landau kinetic equation, we have to expand $f$ in $I_{\mathrm{E}}$ and $n\left(\boldsymbol{r}_{2}, t\right)$ in $I_{\mathrm{MF}}$ in space gradients:

$$
\begin{aligned}
f\left(\boldsymbol{r}_{1} \pm \boldsymbol{\sigma}, \boldsymbol{v}_{2}\right) & =f\left(\boldsymbol{r}_{1}, \boldsymbol{v}_{2}\right) \pm \delta \boldsymbol{\sigma} \cdot \nabla_{1} f\left(\boldsymbol{r}_{1}, \boldsymbol{v}_{2}\right)+\delta^{2} \frac{1}{2} \boldsymbol{\sigma} \boldsymbol{\sigma}: \nabla_{1} \nabla_{1} f\left(\boldsymbol{r}_{1}, \boldsymbol{v}_{2}\right)+\ldots \\
n\left(\boldsymbol{r}_{2}\right) & =n\left(\boldsymbol{r}_{1}\right)+\delta \boldsymbol{r}_{21} \cdot \nabla_{1} n\left(\boldsymbol{r}_{1}\right)+\delta^{2} \frac{1}{2} \boldsymbol{r}_{21} \boldsymbol{r}_{21}: \nabla_{1} \nabla_{1} n\left(\boldsymbol{r}_{1}\right)+\ldots
\end{aligned}
$$

and to substitute into the collision integrals. Also for $g_{2}$ in $I_{\mathrm{E}}$ and $I_{\mathrm{MF}}$ expressions (14)-(16) should be used with substitution of corresponding values of $\boldsymbol{r}_{p}, \boldsymbol{r}_{q}$, and $\boldsymbol{r}_{e}$ in agreement with the table from the previous section. After that equation (1) becomes

$$
\begin{aligned}
\mathcal{D}_{1} f & =I_{\mathrm{E}}^{0}(f, f)+\frac{1}{m} \frac{\partial f}{\partial \boldsymbol{v}_{1}} \cdot \boldsymbol{i}_{\mathrm{MF}}^{0}+\delta\left[I_{\mathrm{E}}^{1}(f, f)+\frac{1}{m} \frac{\partial f}{\partial \boldsymbol{v}_{1}} \cdot \boldsymbol{i}_{\mathrm{MF}}^{1}\right] \\
& +\delta^{2}\left[I_{\mathrm{E}}^{2}(f, f)+\frac{1}{m} \frac{\partial f}{\partial \boldsymbol{v}_{1}} \cdot \boldsymbol{i}_{\mathrm{MF}}^{2}\right]+\ldots+I_{\mathrm{L}}(f, f),
\end{aligned}
$$

where $\mathcal{D}_{1} \equiv\left[\partial_{t}+\boldsymbol{v}_{1} \cdot \nabla_{1}\right]$. Explicit expressions for $I_{\mathrm{E}}^{j}$ are

$$
\begin{aligned}
I_{\mathrm{E}}^{0}(f, h) & =\sigma^{2} \int \mathrm{d} \boldsymbol{v}_{2} \mathrm{~d} \hat{\sigma} \theta\left(\hat{\sigma} \cdot \boldsymbol{v}_{21}\right) \hat{\sigma} \cdot \boldsymbol{v}_{21} \\
& \times\left[g_{2}^{0}\left(+\mid n, \boldsymbol{r}_{1}\right) f^{\prime}(1) h^{\prime}(2)-g_{2}^{0}\left(-\mid n, \boldsymbol{r}_{1}\right) f(1) h(2)\right],
\end{aligned}
$$




$$
\begin{aligned}
& I_{\mathrm{E}}^{1}(f, h)=\sigma^{2} \int \mathrm{d} \boldsymbol{v}_{2} \mathrm{~d} \hat{\sigma} \theta\left(\hat{\sigma} \cdot \boldsymbol{v}_{21}\right) \hat{\sigma} \cdot \boldsymbol{v}_{21} \\
& \times\left\{\left[g_{2}^{0}\left(+\mid n, \boldsymbol{r}_{1}\right) f^{\prime}(1) \nabla_{1} h^{\prime}(2)+g_{2}^{0}\left(-\mid n, \boldsymbol{r}_{1}\right) f(1) \nabla_{1} h(2)\right] \cdot \boldsymbol{\sigma}\right. \\
&\left.+\left[g_{2}^{1}\left(+\mid n, \boldsymbol{r}_{1}\right) f^{\prime}(1) h^{\prime}(2)-g_{2}^{1}\left(-\mid n, \boldsymbol{r}_{1}\right) f(1) h(2)\right]\right\}, \\
& I_{\mathrm{E}}^{2}(f, h)=\sigma^{2} \int \mathrm{d} \boldsymbol{v}_{2} \mathrm{~d} \hat{\sigma} \theta\left(\hat{\sigma} \cdot \boldsymbol{v}_{21}\right) \hat{\sigma} \cdot \boldsymbol{v}_{21} \\
& \quad \times\left\{\left[g_{2}^{0}\left(+\mid n, \boldsymbol{r}_{1}\right) f^{\prime}(1) \nabla_{1} \nabla_{1} h^{\prime}(2)+g_{2}^{0}\left(-\mid n, \boldsymbol{r}_{1}\right) f(1) \nabla_{1} \nabla_{1} h(2)\right]: \frac{1}{2} \boldsymbol{\sigma} \boldsymbol{\sigma}\right. \\
&+\left[g_{2}^{1}\left(+\mid n, \boldsymbol{r}_{1}\right) f^{\prime}(1) \nabla_{1} h^{\prime}(2)+g_{2}^{1}\left(-\mid n, \boldsymbol{r}_{1}\right) f(1) \nabla_{1} h(2)\right] \cdot \boldsymbol{\sigma} \\
&\left.\quad+\left[g_{2}^{2}\left(+\mid n, \boldsymbol{r}_{1}\right) f^{\prime}(1) h^{\prime}(2)-g_{2}^{2}\left(-\mid n, \boldsymbol{r}_{1}\right) f(1) h(2)\right]\right\},
\end{aligned}
$$

where we use the following notations $f^{\prime}(1)=f\left(\boldsymbol{r}_{1}, \boldsymbol{v}_{1}^{\prime}\right), h^{\prime}(2)=h\left(\boldsymbol{r}_{1}, \boldsymbol{v}_{2}^{\prime}\right), f(1)=$ $f\left(\boldsymbol{r}_{1}, \boldsymbol{v}_{1}\right), h(2)=h\left(\boldsymbol{r}_{1}, \boldsymbol{v}_{2}\right), g_{2}^{j}\left( \pm \mid n, \boldsymbol{r}_{1}\right)=g_{2}^{j}\left(\boldsymbol{r}_{1}, \boldsymbol{r}_{1} \pm \boldsymbol{\sigma} \mid n, \boldsymbol{r}_{1}\right)$, and

$$
\begin{aligned}
g_{2}^{0}\left( \pm \mid n, \boldsymbol{r}_{1}\right) & =g_{2}^{0}\left(\sigma \mid n, \boldsymbol{r}_{1}\right)=g_{2}^{\mathrm{eq}}\left(\sigma \mid n, \boldsymbol{r}_{1}\right) \\
g_{2}^{1}\left( \pm \mid n, \boldsymbol{r}_{1}\right) & = \pm \frac{\boldsymbol{\sigma}}{2} \cdot \nabla_{1} n \partial_{n} g_{2}^{0}\left(\sigma \mid n, \boldsymbol{r}_{1}\right) \\
g_{2}^{2}\left( \pm \mid n, \boldsymbol{r}_{1}\right) & =\frac{1}{2} \nabla_{1} \nabla_{1} n:[\nu \mid+(\mu-\nu) \hat{\sigma} \hat{\sigma}] \\
& +\frac{1}{2} \nabla_{1} n \nabla_{1} n:\left[\nu_{2} \mathbf{I}+\left(\mu_{2}-\nu_{2}\right) \hat{\sigma} \hat{\sigma}\right] .
\end{aligned}
$$

equations (29)-(31) are calculated using expressions (14)-(16) and the first column of the table. The factors $\boldsymbol{i}_{\mathrm{MF}}^{j}$ can be calculated immediately using expressions (14)(16) and the third column of the table. They read:

$$
\begin{aligned}
\boldsymbol{i}_{\mathrm{MF}}^{0} & =-\int \mathrm{d} \boldsymbol{r}_{21} \hat{r}_{21} \Phi_{t}^{\prime}\left(r_{21}\right) n\left(\boldsymbol{r}_{1}\right) g_{2}^{0}\left(r_{21} \mid n, \boldsymbol{r}_{1}\right)=0, \\
\boldsymbol{i}_{\mathrm{MF}}^{1} & =-\int \mathrm{d} \boldsymbol{r}_{21} \hat{r}_{21} \Phi_{t}^{\prime}\left(r_{21}\right)\left[\nabla_{1} n\left(\boldsymbol{r}_{1}\right) \cdot \boldsymbol{r}_{21} g_{2}^{0}\left(r_{21} \mid n, \boldsymbol{r}_{1}\right)\right. \\
& \left.+\frac{n\left(\boldsymbol{r}_{1}\right)}{2} \nabla_{1} n\left(\boldsymbol{r}_{1}\right) \cdot \boldsymbol{r}_{21} \partial_{n} g_{2}^{0}\left(r_{21} \mid n, \boldsymbol{r}_{1}\right)\right]=-\nabla_{1} n\left(\boldsymbol{r}_{1}\right)\left[G_{0}+\frac{n\left(\boldsymbol{r}_{1}\right)}{2} G_{1}\right], \\
\boldsymbol{i}_{\mathrm{MF}}^{2} & =-\int \mathrm{d} \boldsymbol{r}_{21} \hat{r}_{21} \Phi_{t}^{\prime}\left(r_{21}\right)\left[\frac{1}{2} \nabla_{1} \nabla_{1} n\left(\boldsymbol{r}_{1}\right) \cdot \boldsymbol{r}_{21} \boldsymbol{r}_{21} g_{2}^{0}\left(r_{21} \mid n, \boldsymbol{r}_{1}\right)\right. \\
& \left.+\nabla_{1} n\left(\boldsymbol{r}_{1}\right) \cdot \boldsymbol{r}_{21} g_{2}^{1}\left(r_{21} \mid n, \boldsymbol{r}_{1}\right)+n\left(\boldsymbol{r}_{1}\right) g_{2}^{2}\left(r_{21} \mid n, \boldsymbol{r}_{1}\right)\right]=0,
\end{aligned}
$$

where

$$
\begin{aligned}
G_{0} & =\frac{4}{3} \int_{\sigma}^{\infty} \mathrm{d} r_{21} r_{21}^{3} \Phi_{t}^{\prime}\left(r_{21}\right) g_{2}^{0}\left(r_{21} \mid n, \boldsymbol{r}_{1}\right), \\
G_{1} & =\frac{4}{3} \int_{\sigma}^{\infty} \mathrm{d} r_{21} r_{21}^{3} \Phi_{t}^{\prime}\left(r_{21}\right) \partial_{n} g_{2}^{0}\left(r_{21} \mid n, \boldsymbol{r}_{1}\right) .
\end{aligned}
$$

Due to equations (32) and (34) $I(f, f)$ changes to:

$$
I(f, f)=I_{\mathrm{E}}^{0}(f, f)+I_{\mathrm{L}}(f, f)+\delta\left[I_{\mathrm{E}}^{1}(f, f)+\frac{1}{m} \frac{\partial f}{\partial \boldsymbol{v}_{1}} \cdot \boldsymbol{i}_{\mathrm{MF}}^{1}\right]+\delta^{2} I_{\mathrm{E}}^{2}(f, f)+\ldots
$$


Here, functions in all the terms are calculated at the same point $\boldsymbol{r}_{1}$ of the space. For such a local form of the total collision integral (35) we can apply the ChapmanEnskog method.

In accordance with this method, the one-particle distribution function is searched in a form of the expansion

$$
f=f^{0}+\varepsilon f^{1}+\varepsilon^{2} f^{2}+\ldots,
$$

$f^{1}$ is the first-order correction linear in the gradients of $n, \boldsymbol{V}$, and $T ; f^{2}$ is the secondorder correction. It consists of the terms which are proportional to the second-order gradients or to double products of the first-order gradients of $n, \boldsymbol{V}$, and $T$. In order to obtain equations for $f^{0}, f^{1}, f^{2}$, we write the kinetic equation (1) in the form

$$
\mathcal{D}_{1} f=\frac{1}{\varepsilon} I(f, f),
$$

substitute equation (35) into its right-hand side, and collect coefficients at the same power of the parameter $\varepsilon$ (the formal parameters $\varepsilon$ and $\delta$ are of the same order). Equations for $f^{j}$ read:

$$
\begin{aligned}
0 & =I_{\mathrm{E}}^{0}\left(f^{0}, f^{0}\right)+I_{\mathrm{L}}\left(f^{0}, f^{0}\right), \\
\left(\mathcal{D}_{1} f\right)^{(0)} & =I_{\mathrm{E}}^{0}\left(f^{0}, f^{1}\right)+I_{\mathrm{E}}^{0}\left(f^{1}, f^{0}\right)+I_{\mathrm{E}}^{1}\left(f^{0}, f^{0}\right)+\frac{1}{m} \frac{\partial f^{0}}{\partial \boldsymbol{v}_{1}} \cdot \boldsymbol{i}_{\mathrm{MF}}^{1} \\
& +I_{\mathrm{L}}\left(f^{0}, f^{1}\right)+I_{\mathrm{L}}\left(f^{1}, f^{0}\right), \\
\left(\mathcal{D}_{1} f\right)^{(1)} & =I_{\mathrm{E}}^{0}\left(f^{0}, f^{2}\right)+I_{\mathrm{E}}^{0}\left(f^{2}, f^{0}\right)+I_{\mathrm{E}}^{0}\left(f^{1}, f^{1}\right)+I_{\mathrm{E}}^{1}\left(f^{0}, f^{1}\right) \\
& +I_{\mathrm{E}}^{1}\left(f^{1}, f^{0}\right)+I_{\mathrm{E}}^{2}\left(f^{0}, f^{0}\right)+\frac{1}{m} \frac{\partial f^{1}}{\partial \boldsymbol{v}_{1}} \cdot \boldsymbol{i}_{\mathrm{MF}}^{1}+I_{\mathrm{L}}\left(f^{0}, f^{2}\right) \\
& +I_{\mathrm{L}}\left(f^{2}, f^{0}\right)+I_{\mathrm{L}}\left(f^{1}, f^{1}\right),
\end{aligned}
$$

where

$$
\begin{aligned}
& \left(\mathcal{D}_{1} f\right)^{(0)}=\left[\partial_{t}^{0}+\boldsymbol{v}_{1} \cdot \nabla_{1}\right] f^{0} \\
& \left(\mathcal{D}_{1} f\right)^{(1)}=\left[\partial_{t}^{0}+\boldsymbol{v}_{1} \cdot \nabla_{1}\right] f^{1}+\partial_{t}^{1} f^{0} .
\end{aligned}
$$

The time derivative is known to be formally expanded in $\varepsilon$ too:

$$
\partial_{t}=\partial_{t}^{0}+\varepsilon \partial_{t}^{1}+\varepsilon^{2} \partial_{t}^{2}+\ldots
$$

The local-equilibrium distribution function $f^{0}=n(m / 2 \pi k T)^{3 / 2} \exp \left\{\frac{-m c_{1}^{2}}{2 k T}\right\}$ is the solution to equation (38). The first correction, which should be sought from equation (39), was found in $[7,8]$. It looks like $f^{1}=f^{0} \phi^{1}$, where

$$
\phi^{1}\left(\boldsymbol{c}_{1}\right)=-\sqrt{\frac{m}{2 k T}} A\left(c_{1}^{2}\right) \boldsymbol{c}_{1} \cdot \nabla_{1} \ln T-\frac{m}{2 k T} B\left(c_{1}^{2}\right)\left(\boldsymbol{c}_{1} \boldsymbol{c}_{1}-\frac{1}{3} c_{1}^{2} \mathbf{l}\right): \nabla_{1} \boldsymbol{V} .
$$

The functions $A$ and $B$ are expressed through $\Omega$-integrals, which, however, besides the hard-sphere part, contain a contribution of the long-range tail $\Phi_{t}\left(r_{21}\right)$.

The second correction $f^{2}$ should be sought from equation (40). 


\section{Contributions of various orders to $P$ and $q$}

Now we shall consider contributions of various orders to the momentum and heat fluxes. According to equation (36) they should be represented as

$$
\mathrm{P}=\mathrm{P}^{0}+\varepsilon \mathrm{P}^{1}+\varepsilon^{2} \mathrm{P}^{2}+\ldots, \quad \boldsymbol{q}=\boldsymbol{q}^{0}+\varepsilon \boldsymbol{q}^{1}+\varepsilon^{2} \boldsymbol{q}^{2}+\ldots
$$

Kinetic parts are

$$
\left(\begin{array}{c}
\mathrm{P}^{k(j)} \\
\boldsymbol{q}^{k(j)}
\end{array}\right)=\int \mathrm{d} \boldsymbol{v}_{1} f^{j}\left(x_{1}, t\right)\left(\begin{array}{c}
m \boldsymbol{c}_{1} \boldsymbol{c}_{1} \\
\frac{m}{2} c_{1}^{2} \boldsymbol{c}_{1}
\end{array}\right)
$$

In order to find the collisional contributions it is necessary to expand the function $f_{2}$ in equation (9) in $\lambda$ and afterwards this expansion should be integrated over $\lambda$. Collecting terms of the same order with respect to the parameters $\varepsilon$ and $\delta$, we find:

$$
\left(\begin{array}{l}
\operatorname{Phs}(0) \\
\operatorname{Phs}(1) \\
\operatorname{Phs}(2)
\end{array}\right)=\frac{\sigma^{3}}{2} \int \mathrm{d} \boldsymbol{v}_{1} \mathrm{~d} \boldsymbol{v}_{2} \mathrm{~d} \hat{\sigma} \theta\left(\hat{\sigma} \cdot \boldsymbol{v}_{21}\right) \hat{\sigma} \cdot \boldsymbol{v}_{21} m\left[\boldsymbol{v}_{1}^{\prime}-\boldsymbol{v}_{1}\right] \hat{\sigma}\left(\begin{array}{l}
F_{2}^{(0)} \\
F_{2}^{(1)} \\
F_{2}^{(2)}
\end{array}\right),
$$

where

$$
\begin{aligned}
& F_{2}^{(0)}=g_{2}^{0}\left( \pm \mid n, \boldsymbol{r}_{1}\right) f^{0}(1) f^{0}(2), \\
& F_{2}^{(1)}=g_{2}^{0}\left( \pm \mid n, \boldsymbol{r}_{1}\right) f^{0}(1) f^{0}(2)\left[\phi^{1}(1)+\phi^{1}(2)+\frac{\boldsymbol{\sigma}}{2} \cdot \nabla_{1} \ln \frac{f^{0}(1)}{f^{0}(2)}\right] \\
& F_{2}^{(2)}=f^{0}(1) f^{0}(2)\left(g _ { 2 } ^ { 0 } ( \pm | n , \boldsymbol { r } _ { 1 } ) \left\{\phi^{2}(1)+\phi^{2}(2)+\phi^{1}(1) \phi^{1}(2)\right.\right. \\
& \quad+\frac{1}{2} \boldsymbol{\sigma} \cdot\left[\left(\phi^{1}(1)+\phi^{1}(2)\right) \nabla_{1} \ln \frac{f^{0}(1)}{f^{0}(2)}+\nabla_{1} \phi^{1}(1)-\nabla_{1} \phi^{1}(2)\right] \\
& \left.\quad+\frac{1}{6} \boldsymbol{\sigma} \boldsymbol{\sigma}:\left[\frac{\nabla_{1} \nabla_{1} f^{0}(1)}{f^{0}(1)}+\frac{\nabla_{1} \nabla_{1} f^{0}(2)}{f^{0}(2)}-\nabla_{1} \ln f^{0}(1) \nabla_{1} \ln f^{0}(2)\right]\right\} \\
& \quad+\frac{1}{12} \tau_{0} \nabla_{1} n \cdot \boldsymbol{\sigma} \boldsymbol{\sigma} \cdot \nabla_{1} \ln \left(f^{0}(1) f^{0}(2)\right) \\
& \quad+\frac{1}{2} \nabla_{1} \nabla_{1} n:\left[\nu \mathbf{l}+(\mu-\nu) \hat{\sigma} \hat{\sigma}-\frac{1}{6} \tau_{0} \boldsymbol{\sigma} \boldsymbol{\sigma}\right] \\
& \left.\quad+\frac{1}{2} \nabla_{1} n \nabla_{1} n:\left[\nu_{2} \mathbf{I}+\left(\mu_{2}-\nu_{2}\right) \hat{\sigma} \hat{\sigma}-\frac{1}{6} \partial_{n} \tau_{0} \boldsymbol{\sigma} \boldsymbol{\sigma}\right]\right)
\end{aligned}
$$

with $\phi^{2}(l)=f^{2}(l) / f^{0}(l), l=1,2$. Expressions for $\boldsymbol{q}^{h s(j)}$ have got the same structure as (43), but $m\left[\boldsymbol{v}_{1}^{\prime}-\boldsymbol{v}_{1}\right]$ should be substituted by $m\left[c_{1}^{\prime 2}-c_{1}^{2}\right] / 2$.

The $\mathrm{P}^{t(j)}$ contributions can be found similar to the collision ones, except that the function $n_{2}$ in equation (8) should be expanded and integrated:

$$
\left(\begin{array}{l}
\mathrm{P}^{t(0)} \\
\mathrm{P}^{t(1)} \\
\mathrm{P}^{t(2)}
\end{array}\right)=-\frac{1}{2} \int \mathrm{d} \boldsymbol{s} \boldsymbol{s} \hat{s} \Phi_{t}^{\prime}(s)\left(\begin{array}{c}
n^{2} g_{2}^{0}\left(s \mid n, \boldsymbol{r}_{1}\right) \\
0 \\
N_{2}^{(2)}
\end{array}\right)
$$


where

$$
\begin{aligned}
N_{2}^{(2)}= & g_{2}^{0}\left(s \mid n, \boldsymbol{r}_{1}\right)\left[\frac{1}{3} n \nabla_{1} \nabla_{1} n-\frac{1}{6} \nabla_{1} n \nabla_{1} n\right]: s \boldsymbol{s}+\frac{1}{6} n \nabla_{1} n \nabla_{1} n: \boldsymbol{s} \boldsymbol{s} \tau_{0}(s) \\
& +n^{2}\left(\frac{1}{2} \nabla_{1} \nabla_{1} n:\left[\nu \mathbf{l}+(\mu-\nu) \hat{s} \hat{s}-\frac{1}{6} \tau_{0}(s) \boldsymbol{s s}\right]\right. \\
& \left.+\frac{1}{2} \nabla_{1} n \nabla_{1} n:\left[\nu_{2} \mathbf{I}+\left(\mu_{2}-\nu_{2}\right) \hat{s} \hat{s}-\frac{1}{6} \partial_{n} \tau_{0}(s) \boldsymbol{s s}\right]\right) .
\end{aligned}
$$

Using expressions (46), (48) for $F_{2}^{(2)}$ and $N_{2}^{(2)}$, one can find $\mathrm{P}^{h s(2)}, \boldsymbol{q}^{h s(2)}$, and $\mathrm{P}^{t(2)}$. The last term can be calculated at once, performing integration over orientations of $s$, whereas $F_{2}^{(2)}$ contains the function $\phi^{2}=f^{2} / f^{0}$ being unknown so far.

\section{Conclusive remarks}

So, considering the second-order approximation to the one-particle distribution function in the Chapman-Enskog method we have obtained the following results. We found the second-order term (16) of the gradient expansion for the pair distribution function in the approximation equation (12) in the general case. This term greatly differs from the corresponding term for the equilibrium pair distribution function. The second-order contributions to the momentum and heat fluxes were found exploiting this expression.

Analyzing the explicit expressions for $\boldsymbol{i}_{\mathrm{MF}}^{j}$ the general form equation (40) of the equation for the second-order correction was written. To simplify this equation, it is necessary to express the time derivatives $\partial_{t}^{0}$ and $\partial_{t}^{1}$ of $n, \boldsymbol{V}, T$ in $\left(\mathcal{D}_{1} f\right)^{1}$ through the gradients of these quantities using the following equations:

$$
\begin{gathered}
\partial_{t}^{0} n=-\nabla_{1} \cdot(n \boldsymbol{V}), \\
\partial_{t}^{0} \boldsymbol{V}=-\boldsymbol{V} \cdot \nabla_{1} \boldsymbol{V}-\frac{1}{\rho} \nabla_{1} P^{0}, \\
\partial_{t}^{0} T=-\boldsymbol{V} \cdot \nabla_{1} T-\frac{2}{3 k n} P_{*}^{0} \nabla_{1} \cdot \boldsymbol{V}, \\
\partial_{t}^{1} n=0 \\
\partial_{t}^{1} \boldsymbol{V}=\frac{1}{\rho}\left[\nabla_{1}\left(\kappa \nabla_{1} \cdot \boldsymbol{V}\right)+2 \nabla_{1} \cdot(\eta \mathrm{S})\right], \\
\partial_{t}^{1} T=\frac{2}{3 k n}\left[\nabla_{1} \cdot\left(\lambda \nabla_{1} T\right)+\kappa\left(\nabla_{1} \cdot \boldsymbol{V}\right)^{2}+2 \eta \mathrm{S}: \mathrm{S}\right],
\end{gathered}
$$

where

$$
\begin{gathered}
\mathrm{P}^{0}=\mathrm{I} P^{0}=\mathrm{I}\left(P^{k(0)}+P^{h s(0)}+P^{t(0)}\right), \quad \boldsymbol{q}^{0}=0, \\
P^{k(0)}+P^{h s(0)}=\left[1+\frac{2}{3} \pi \sigma^{3} n g_{2}^{0}\left(\sigma \mid n, \boldsymbol{r}_{1}\right)\right] n k T, \\
P^{t(0)}=-\frac{2}{3} \pi n^{2} \int_{\sigma}^{\infty} \mathrm{d} s s^{3} \Phi_{t}^{\prime}(s) g_{2}^{0}\left(s \mid n, \boldsymbol{r}_{1}\right), \\
\mathrm{P}^{1}=\mathrm{P}^{k(1)}+\mathrm{P}^{h s(1)}=-\kappa \mathrm{I} \nabla_{1} \cdot \boldsymbol{V}-2 \eta \mathrm{S}, \quad \boldsymbol{q}^{1}=-\lambda \nabla_{1} T .
\end{gathered}
$$

Transport coefficients $\kappa, \eta$, and $\lambda$ (bulk and shear viscosities and thermal conductivity) depend on $\boldsymbol{r}_{1}$ and $t$ through $n$ and $T$, whereas in the case of the Boltzmann kinetic equation $\kappa$ is absent, and $\eta$ and $\lambda$ depend on temperature only. 
Besides, in order to simplify equation (40) it is necessary to find all the integrals in its right-hand side which do not contain $f^{2}$. Then we shall obtain an integral equation, the left-hand side of which will be expressed through the second gradients and double products of the first gradients of $n, \boldsymbol{V}$ and $T$. The correction $f^{2}$ should be sought from that equation. It can be solved with the same method, which is used to find the correction $f^{1}$.

Since the hydrodynamic quantities $n, \boldsymbol{V}, T$ are completely determined by the zeroth approximation $f^{0}$ of the one-particle distribution function, the following additional conditions should be imposed for $f^{2}$ :

$$
\int \mathrm{d} \boldsymbol{v}_{1} \psi\left(\boldsymbol{v}_{1}\right) f^{2}\left(x_{1}, t\right)=0,
$$

where $\psi\left(\boldsymbol{v}_{1}\right)=\left\{1, m \boldsymbol{v}_{1}, m v_{1}^{2} / 2\right\}$.

Notice that our results reduce to the second-order approximation for the kinetic equation of KMFT, when $I_{\mathrm{L}}$ is neglected in equation (1). When both $I_{\mathrm{L}}$ and $I_{\mathrm{MF}}$ are ignored, we obtain exact results of the second-order approximation for the modified Enskog equation (RET). The theory can be also applied to the system with a continuous potential alone (without the hard-sphere part), but in the low-density case only.

\section{A. Details of calculating $g_{2}^{j}$}

Here we give some details of the derivation of equation (15), (16) from (13). It consists in a transition from the Cartesian system of coordinates to the bipolar system.

Let us choose the Cartesian system of coordinates with the origin in $\boldsymbol{r}_{p}$ and with the axis $O z$ along the vector $\boldsymbol{r}_{q p}$. Transition to the bipolar system with poles in $\boldsymbol{r}_{p}$ and $\boldsymbol{r}_{q}$ is performed using the following formulae:

$$
r_{s p}^{x}=\frac{1}{2 r} \xi(r, s, t) \cos \varphi, \quad r_{s p}^{y}=\frac{1}{2 r} \xi(r, s, t) \sin \varphi, \quad r_{s p}^{z}=\frac{1}{2 r}\left(s^{2}-t^{2}+r^{2}\right),
$$

where $r=r_{q p}, s=r_{s p}, t=r_{s q}, \xi(r, s, t)=\sqrt{\left[t^{2}-(s-r)^{2}\right]\left[(s+r)^{2}-t^{2}\right]}$, and $\varphi$ is an azimuthal angle. The coordinates of the bipolar reference system are $s \in[0 ;+\infty)$, $t \in[|r-s| ; r+s], \varphi \in[0 ; 2 \pi]$, but $r$ is an inner parameter of this reference system $[6]$.

Let us consider the term $\sim \delta$ in equation (13). Since $\nabla_{e} n\left(\boldsymbol{r}_{e}\right)$ does not depend on $\boldsymbol{r}_{s}$, we can take this gradient out of the integral and write it using the expression

$$
\boldsymbol{r}_{s e}=\boldsymbol{r}_{s p}+\boldsymbol{r}_{p e}
$$

in the form

$\int \mathrm{d} \boldsymbol{r}_{s} H^{1}\left(\boldsymbol{r}_{p}, \boldsymbol{r}_{q} ; \boldsymbol{r}_{s} \mid n, \boldsymbol{r}_{e}\right)\left[\boldsymbol{r}_{s p}+\boldsymbol{r}_{p e}\right]=\int \mathrm{d} \boldsymbol{r}_{s} H^{1}\left(\boldsymbol{r}_{p}, \boldsymbol{r}_{q} ; \boldsymbol{r}_{s} \mid n, \boldsymbol{r}_{e}\right) \boldsymbol{r}_{s p}+\partial_{n} g_{2}^{\mathrm{eq}}\left(\boldsymbol{r}_{p}, \boldsymbol{r}_{q} \mid n, \boldsymbol{r}_{e}\right) \boldsymbol{r}_{p e}$, 
where $\boldsymbol{r}_{i j}=\boldsymbol{r}_{i}-\boldsymbol{r}_{j}$ and the following equivalence has been taken into account:

$$
\int \mathrm{d} \boldsymbol{r}_{s} H^{1}\left(\boldsymbol{r}_{p}, \boldsymbol{r}_{q} ; \boldsymbol{r}_{s} \mid n, \boldsymbol{r}_{e}\right)=\partial_{n} g_{2}^{\mathrm{eq}}\left(\boldsymbol{r}_{p}, \boldsymbol{r}_{q} \mid n, \boldsymbol{r}_{e}\right)
$$

Note that the function $H^{1}$ is symmetric under interchange $\boldsymbol{r}_{p \leftarrow} \rightarrow \boldsymbol{r}_{q}$, and depends on relative distances: $H^{1}\left(r_{q p}, r_{s p}, r_{s q} \mid n, \boldsymbol{r}_{e}\right)$. The first term of the right-hand side of equation (57) in the bipolar system reads:

$$
\int_{0}^{\infty} \mathrm{d} s \int_{|r-s|}^{r+s} \mathrm{~d} t \int_{0}^{2 \pi} \mathrm{d} \varphi J H^{1}(r, s, t) \frac{\xi(r, s, t)}{2 r}\left(\begin{array}{c}
\cos \varphi \\
\sin \varphi \\
\omega
\end{array}\right)
$$

with $\omega=\left(s^{2}-t^{2}+r^{2}\right) / \xi, J$ is the transformation Jacobian and equals $s t / r$. After the integration over $\varphi$, only the $z$-component remains. Using the symmetric property of $H^{1}$ under interchange $\vec{\leftarrow} t$ and the equality of rights of these coordinates of the bipolar reference system one can show that $s^{2}-t^{2}$ in $\omega$ does not contribute to the integral. The final result can be written in the Cartesian system as

$$
\int \mathrm{d} \boldsymbol{r}_{s p} H^{1}\left(\boldsymbol{r}_{p}, \boldsymbol{r}_{q} ; \boldsymbol{r}_{s} \mid n, \boldsymbol{r}_{e}\right) \boldsymbol{r}_{s p}=\frac{1}{2} \boldsymbol{r}_{q p} \partial_{n} g_{2}^{\mathrm{eq}}\left(\boldsymbol{r}_{p}, \boldsymbol{r}_{q} \mid n, \boldsymbol{r}_{e}\right)
$$

Substituting it into equation (57) we get expression (15) for $g_{2}^{1}$.

The first term of equation (13) which is $\sim \delta^{2}$ can be found in the same way. After substituting (56) into it, the corresponding integral becomes

$$
\int \mathrm{d} \boldsymbol{r}_{s p} H^{1}\left(\boldsymbol{r}_{p}, \boldsymbol{r}_{q} ; \boldsymbol{r}_{s} \mid n, \boldsymbol{r}_{e}\right)\left[\boldsymbol{r}_{s p} \boldsymbol{r}_{s p}+\boldsymbol{r}_{s p} \boldsymbol{r}_{p e}+\boldsymbol{r}_{p e} \boldsymbol{r}_{s p}+\boldsymbol{r}_{p e} \boldsymbol{r}_{p e}\right] .
$$

The second and third terms in the square brackets may be found using equation (59). To find the forth term, equation (58) should be used. The first term of equation (60) in the bipolar system reads:

$$
\int_{0}^{\infty} \mathrm{d} s \int_{|r-s|}^{r+s} \mathrm{~d} t \int_{0}^{2 \pi} \mathrm{d} \varphi J H^{1}(r, s, t) \frac{\xi^{2}}{4 r^{2}}\left(\begin{array}{ccc}
\cos ^{2} \varphi & \cos \varphi \sin \varphi & \omega \cos \varphi \\
\sin \varphi \cos \varphi & \sin ^{2} \varphi & \omega \sin \varphi \\
\omega \cos \varphi & \omega \sin \varphi & \omega^{2}
\end{array}\right)
$$

After integrating over $\varphi$ only diagonal elements of the tensor remain:

$$
\int_{0}^{\infty} \mathrm{d} s \int_{|r-s|}^{r+s} \mathrm{~d} t \int_{0}^{2 \pi} \mathrm{d} \varphi J H^{1}(r, s, t) \frac{\xi^{2}}{4 r^{2}}\left(\begin{array}{ccc}
\pi & 0 & 0 \\
0 & \pi & 0 \\
0 & 0 & 2 \pi \omega^{2}
\end{array}\right)=\nu \mathbf{l}+(\mu-\nu) \mathbf{I}_{3},
$$

where $\mathrm{I}$ is the unit tensor, and

$$
\mathrm{I}_{3}=\left(\begin{array}{lll}
0 & 0 & 0 \\
0 & 0 & 0 \\
0 & 0 & 1
\end{array}\right)
$$


The coefficients

$$
\nu=\frac{\pi}{4 r^{2}} \int_{0}^{\infty} \mathrm{d} s \int_{|r-s|}^{r+s} \mathrm{~d} t J H^{1}(r, s, t) \xi^{2}, \quad \mu=\frac{\pi}{2 r^{2}} \int_{0}^{\infty} \mathrm{d} s \int_{|r-s|}^{r+s} \mathrm{~d} t J H^{1}(r, s, t) \omega^{2} \xi^{2}
$$

can be written in the form of equations (17), (18) using the symmetric properties of $H^{1}$. Notice that the tensor $I_{3}$ is of the form equation (62) only in the chosen Cartesian system of coordinates, in which vector $\boldsymbol{r}_{q p}=\left(0,0, r_{q p}\right)$. In an arbitrary Cartesian reference system, "in which $\boldsymbol{r}_{q p}=\left(r_{q p}^{x}, r_{q p}^{y}, r_{q p}^{z}\right)$ ", the tensor $\mathrm{I}_{3}$ equals $\hat{r}_{q p} \hat{r}_{q p}$. Combining equation (61) and equation (60) we obtain the first two terms of expression (16) for $g_{2}^{2}$.

Now consider the second term of equation (13) being $\sim \delta^{2}$. The corresponding integral of $H^{2}$ can be written similar to equation (60):

$$
\int \mathrm{d} \boldsymbol{r}_{s p} \mathrm{~d} \boldsymbol{r}_{v p} H^{2}\left(\boldsymbol{r}_{p}, \boldsymbol{r}_{q} ; \boldsymbol{r}_{s}, \boldsymbol{r}_{v} \mid n, \boldsymbol{r}_{e}\right)\left[\boldsymbol{r}_{s p} \boldsymbol{r}_{v p}+\boldsymbol{r}_{s p} \boldsymbol{r}_{p e}+\boldsymbol{r}_{p e} \boldsymbol{r}_{v p}+\boldsymbol{r}_{p e} \boldsymbol{r}_{p e}\right] .
$$

The second, third and forth terms in square brackets may be evaluated using

$$
\int \mathrm{d} \boldsymbol{r}_{v p} H^{2}\left(\boldsymbol{r}_{p}, \boldsymbol{r}_{q} ; \boldsymbol{r}_{s}, \boldsymbol{r}_{v} \mid n, \boldsymbol{r}_{e}\right)=H^{1}\left(\boldsymbol{r}_{p}, \boldsymbol{r}_{q} ; \boldsymbol{r}_{s} \mid n, \boldsymbol{r}_{e}\right)
$$

and formulae (58), (59). We get for them

$$
\partial_{n} \partial_{n} g_{2}^{\mathrm{eq}}\left(\boldsymbol{r}_{p}, \boldsymbol{r}_{q} \mid n, \boldsymbol{r}_{e}\right)\left[\frac{1}{2}\left(\boldsymbol{r}_{q p} \boldsymbol{r}_{p e}+\boldsymbol{r}_{p e} \boldsymbol{r}_{q p}\right)+\boldsymbol{r}_{p e} \boldsymbol{r}_{p e}\right] .
$$

Before considering the first term in square brackets of equation (63), we discuss symmetric properties of $H^{2}\left(\boldsymbol{r}_{p}, \boldsymbol{r}_{q} ; \boldsymbol{r}_{s}, \boldsymbol{r}_{v} \mid n, \boldsymbol{r}_{e}\right)$. It depends only on relative distances $\left|\boldsymbol{r}_{i j}\right|$, where indices $i, j=p, q, s, v: H^{2}=H^{2}\left(r_{q p},\left[\begin{array}{l}r_{s p}, r_{v p} \\ r_{s q}, r_{v q}\end{array}, r_{s v} \mid n, \boldsymbol{r}_{e}\right)\right.$. Arguments $\boldsymbol{r}_{p}$ and $\boldsymbol{r}_{q}$ are equal in rights as well as $\boldsymbol{r}_{s}$ and $\boldsymbol{r}_{v}$. That is why $H^{2}$ is symmetric under interchanges of $\boldsymbol{r}_{p_{\leftarrow}} \rightarrow \boldsymbol{r}_{q}$ and $\boldsymbol{r}_{s} \rightarrow \boldsymbol{r}_{v}$. Dependence of $H^{2}$ on $r_{s v}$ can be reduced to dependence of $H^{2}$ on the difference of the azimuthal angles $\varphi_{1}-\varphi$.

Now let's observe the first term of equation (63). It can be written in the bipolar system as

$$
\begin{aligned}
\int_{0}^{\infty} \mathrm{d} s \int_{|r-s|}^{r+s} \mathrm{~d} t \int_{0}^{\infty} \mathrm{d} s_{1} \int_{\left|r-s_{1}\right|}^{r+s_{1}} \mathrm{~d} t_{1} \int_{0}^{2 \pi} \mathrm{d} \varphi \int_{0}^{2 \pi} \mathrm{d} \varphi_{1} J J_{1} H^{2}\left(r,\left[\begin{array}{c}
s, s_{1} \\
t, t_{1}
\end{array}\right], \varphi_{1}-\varphi \mid n, \boldsymbol{r}_{e}\right) \\
\times \frac{\xi(r, s, t) \xi\left(r, s_{1}, t_{1}\right)}{4 r^{2}}\left(\begin{array}{ccc}
\cos \varphi \cos \varphi_{1} & \cos \varphi \sin \varphi_{1} & \cos \varphi \omega_{1} \\
\sin \varphi \cos \varphi_{1} & \sin \varphi \sin \varphi_{1} & \sin \varphi \omega_{1} \\
\omega \cos \varphi_{1} & \omega \sin \varphi_{1} & \omega \omega_{1}
\end{array}\right) .
\end{aligned}
$$

One can perform integration in equation (64) over one of the angles $\varphi$ or $\varphi_{1}$. Carrying out the transition from variables $\left(\varphi, \varphi_{1}\right)$ to $(\varphi, \chi)$, with $\chi=\varphi_{1}-\varphi$ and integrating the tensor in parentheses of equation (64) over $\varphi$, we obtain it as follows:

$$
\pi\left(\begin{array}{ccc}
\cos \chi & \sin \chi & 0 \\
-\sin \chi & \cos \chi & 0 \\
0 & 0 & 2 \omega \omega_{1}
\end{array}\right)
$$


The function $H^{2}$ is even with respect to $\chi$. Thus non-diagonal elements of the tensor (with $\sin \chi$ ) vanish. Equation (64) becomes similar to the previous case:

$$
\nu_{2} \mathbf{I}-(\mu-\nu) \mathbf{I}_{3}
$$

where the coefficients $\mu_{2}, \nu_{2}$ may be expressed by equations (19), (20) using the symmetric properties of $H^{2}$ mentioned above.

\section{References}

1. Ferziger J.H., Kaper H.G. Mathematical Theory of Transport Processes in Gases. Amsterdam, North-Holland, 1972.

2. van Beijeren H., Ernst M.H. The modified Enskog equation. // Physica (Utrecht), 1973, vol. 68, No. 3, p. 437-456.

3. Zubarev D.N., Morozov V.G., Omelyan I.P., Tokarchuk M.V. On kinetic equations for dense gases and liquids. // Teor. Mat. Fiz., 1991, vol. 87, No. 1, p. 113-129 (in Russian).

4. Karkheck J., Stell G. Kinetic mean-field theories. // J. Chem. Phys., 1981, vol. 75, No. 3, p. 1475-1787.

5. Zubarev D.N., Morozov V.G. Formulation of boundary conditions for the Bogoliubov hierarchy with allowance for local conservation laws. // Teor. Mat. Fiz., 1984, vol. 60, No. 2, p. 270-279 (in Russian).

6. Balescu R. Equilibrium and Non-equilibrium Statistical Mechanics. New York, WileyInterscience, 1975.

7. Tokarchuk M.V., Omelyan I.P., Kobryn O.E. Enskog-Landau kinetic equation. Calculation of the transport coefficients for charged hard spheres model. Preprint of Institute for Condensed Matter Physics, ICMP-92-22U, Lviv, 1992 (in Ukrainian).

8. Kobryn A.E., Morozov V.G., Omelyan I.P., Tokarchuk M.V. Enskog-Landau kinetic equation. Calculation of the transport coefficients for charged hard spheres. // Physica A, 1996, vol. 230, No 1\&2, p. 189-201. 


\title{
Загальні вирази наближення другого порядку методу Чепмена-Енскога для кінетичного рівняння Енскога-Ландау
}

\author{
М.В.Токарчук, Й.А.Гуменюк \\ Інститут фізики конденсованих систем НАН України, \\ 79011 Львів, вул. Свєнціцького, 1 \\ Отримано 13 жовтня 2000 р., в остаточному вигляді - \\ 4 грудня 2000 р.
}

Розглядається наближення другого порядку методу Чепмена-Енскога для кінетичного рівняння Енскога-Ландау. Представлено точні вирази для парної функції розподілу неоднорідної системи твердих кульок до другого порядку по градієнтах включно. Отримано загальне рівняння для поправки другого порядку до одночастинкової функції розподілу. Знайдено відповідні внески до потоків імпульсу і тепла.

Ключові слова: кінетичне рівняння, метод Чепмена-Енскога, поправка другого порядку

PACS: 05.20.Dd, 05.60.-k, 52.25. Fi 